\title{
Riesgo aumentado de cáncer de mama, ovárico y contralateral para portadoras de mutación BRCA1 y BRCA2
}

\author{
Increased risk of breast, ovarian and contralateral cancer for BRCA1 and BRCA2 mutation carriers
}

\section{Comentado de:}

Kuchenbaecker KB et al. JAMA. 2017; 317(23):2402-2416. PMID: $28632866^{1}$

\section{Objetivo}

Estimar el riesgo específico para la edad de cáncer de mama, ovario y cáncer de mama contralateral para portadoras de las mutaciones BRCA1 y BRCA2 y evaluar la modificación del riesgo por la historia familiar.

\section{Diseño, lugar y pacientes}

Estudio de cohorte prospectiva, multicéntrico, internacional.

Se incluyeron 9.856 mujeres, 6.036 de ellas portadoras de la mutación BRCA1 y 3.820 , de BRCA2 (5.046 no afectadas y 4.890 con cáncer de mama, ovario o ambos, al inicio del estudio).

Las participantes fueron reclutadas entre 1997 y 2011 a través del International BRCA1/2 Carrier Cohort Study (IBCCS), el Breast Cancer Family Registry (BCFR) y el Kathleen Cuningham Foundation Consortium para la investigación de cancer de mama familiar (kConFab).

La mayoría fueron grandes estudios nacionales de Reino Unido (EMBRACE), los Países Bajos (HEBON) y Francia (GENEPSO).
La mediana de seguimiento fue de 5 años, finalizando en diciembre de 2013

\section{Medición de resultados principales}

Incidencias anuales, índices de incidencia estandarizados y riesgos acumulativos de cáncer de mama, de ovario y de mama contralateral.

\section{Resultados principales}

Se dividieron en tres grupos, 3.886 mujeres para el análisis de cáncer de mama, 5.066 mujeres para el análisis de cáncer de ovario y 2.213 mujeres para el análisis de cáncer de mama contralateral. Fueron diagnosticadas 426 con cáncer de mama, 109 con cáncer de ovario y 245 con cáncer de mama contralatera durante el seguimiento. Los resultados principales se muestran en la Tabla 1.

En el cáncer de mama el riesgo aumenta con el número creciente de familiares de primer y segundo grado diagnosticados con cáncer de mama tanto para BRCA1 (Hazar Ratio [HR] para 2 vs 0 familiares afectados: 1,99; Intervalo de Confianza [IC] $95 \%$ $1,41$ a 2,$82 ; \mathrm{P}<0,001)$ y portadores BRCA2 (HR: 1,91 ; IC $95 \%$, $1,08$ a 3,$37 ; P=0,02)$.

Tabla 1. Riesgo acumulado de cáncer de mama, ovario y mama contralateral para mujeres portadoras de las mutaciones BRCA 1 y BRCA 2. Notas: *Riesgos acumulados a los 80 años; ${ }^{* *}$ Riesgo acumulado 20 años posteriores al primer diagnóstico.

\begin{tabular}{|c|c|c|}
\hline Localización del cáncer & $\begin{array}{c}\text { Portadoras BRCA1 }(n=6036) \\
\%(\text { IC } 95 \%)\end{array}$ & $\begin{array}{c}\text { Portadoras BRCA2 }(n=3820) \\
\%(\text { IC } 95 \%)\end{array}$ \\
\hline Cáncer de mama * & 72 (65\% a $79 \%)$ & $69(61 \%$ a $77 \%)$ \\
\hline Cáncer de ovario * & 44 (36\% a $53 \%)$ & 17 (11\% a $25 \%)$ \\
\hline Cáncer de mama contralateral ** & $40(35 \%$ a $45 \%)$ & $26(20 \%$ a $33 \%)$ \\
\hline
\end{tabular}

\section{Conclusiones}

Estos hallazgos proporcionan estimaciones del riesgo de cáncer basado en el estado de la portadora de la mutación BRCA1 y BRCA2 mediante la recopilación prospectiva de datos y demuestran la importancia potencial de la historia familiar.

Fuente de financiamiento/ Conflicto de interés de los autores: No referida.

\section{Comentario}

Este estudio describe la incidencia y el riesgo acumulado para portadoras de BRCA1 y BRCA2 de desarrollar cáncer de mama, de ovario y de mama contralateral en una cohorte prospectiva numerosa.

Uno de los hallazgos más interesantes es que, incluso dentro de las portadoras conocidas de las mutaciones, aquellas con historia familiar presentan un aumento en la incidencia de enfermedad oncológica, lo cual podría explicarse por la multicausalidad (genética y no genética) que se agregan en las familias, asociadas al desarrollo de cáncer.

Los resultados de esta investigación coinciden con los de otras cohortes de tipo retrospectiva ${ }^{2}$ y refuerza la recomendación de la USPTF, por la cual, ante pacientes con historia de familiares de primer grado con cáncer de mama, ovario, trompa o peritoneo, el médico de atención primaria debería aplicar alguna herramienta de cálculo de riesgo de ser portadora de las mutaciones BRCA1 y BRCA2, y ante un resultado positivo, derivarla para asesoramiento genético (recomendación tipo $\mathrm{B})^{3}$. 


\section{Conclusiones de la comentadora}

Si bien podría cuestionarse la aplicabilidad de estos resultados como médicos de atención primaria, ya que trabajamos habitualmente con mujeres que desconocen su estatus de portadoras y quienes suelen referirse al especialista ante sospecha de enfermedades oncológicas de agregación familiar, resulta importante conocer el aumento del riesgo de presentar enfermedades malignas en las pacientes que tienen mutaciones de los genes BRCA 1 y 2 por ser los más frecuentes dentro de los sindromes de cáncer de mama hereditario, para acompañarlas en los procesos de toma de decisiones vinculadas con su salud.

Mariel Natalia Mandel [ Servicio de Medicina Familiar y Comunitaria, Hospital Italiano de Buenos Aires mariel.mandel@hospitalitaliano.org.ar ]

Mandel MN Riesgo aumentado de cáncer de mama, ovárico y contralateral para portadoras de mutación BRCA1 y BRCA2 . Evid Actual Pract Ambul. 2019;22(3):e001091. Comentado de: Kuchenbaecker KB et al. Risks of Breast, Ovarian, and Contralateral Breast Cancer for BRCA1 and BRCA2 Mutation Carriers. JAMA. 2017; 317(23):2402-2416. PMID 28632866

\section{Referencias}

1. Kuchenbaecker KB, Hopper JL, Barnes DR, Phillips KA, Mooij TM, Roos-Blom MJ, et al. Risks of breast, ovarian, and contralateral breast cancer for BRCA1 and BRCA2 mutation carriers. JAMA - Journal of the American Medical Association. 2017;317(23):2402-2416. Available from: 10.1001/ jama.2017.7112.

2. Ponder B, Pharoah PDP, Ponder BAJ, Lipscombe JM, Basham V, Gregory J, et al. Prevalence and penetrance of BRCA1 and BRCA2 mutations in a population-based series of breast cancer cases. British Journal of Cancer. 2000;83(10):1301-1308. Available from: 10.1054/bjoc.2000.1407.

3. Owens DK, Davidson KW, Krist AH, Barry MJ, Cabana M, Caughey AB, et al. Risk Assessment, Genetic Counseling, and Genetic Testing for BRCA -Related Cancer. JAMA. 2019;322(7):652. Available from: 10.1001/jama.2019.10987. 